O. WIESEAW KIWIOR OCD

Wydział Prawa Kanonicznego

Uniwersytet Kardynała Stefana Wyszyńskiego w Warszawie

ORCID: 0000-0001-7378-1256

\title{
LA REVISIONE DEL DIRITTO PROPRIO DEI CARMELITANI SCALZI AL TEMPO DI OGGI
}

Contenuto: Introduzione. - 1. L'articolazione del diritto dei singoli istituti religiosi. - 1.1. Le Costituzioni e il loro scopo. - 1.1.1. Il contenuto delle Costituzioni. - 1.1.2. I criteri della redazione delle Costituzioni. - 1. 2. Altri codici. - 2. L'autorità competente per l'approvazione e la modifica del diritto proprio. - 2.1. Le Costituzioni. - 2.2. Altri codici. - 3. Il ruolo delle Costituzioni nel rinnovamento della vita religiosa ed il loro aggiornamento. - 4. Il percorso dell'elaborazione del diritto proprio OCD vigente. - Conclusione

\section{Introduzione}

Il Codice di Diritto Canonico riconosce ai singoli istituti religiosi, in quanto godono di un dono particolare dello Spirito Santo, "una giusta autonomia di vita, specialmente di governo, mediante la quale possano valersi nella Chiesa di una propria disciplina e conservare integro il proprio patrimonio". Detta autonomia "è «giusta» in quanto è determinata e protetta dal diritto comune e proprio" 2 . Essa però "non

\footnotetext{
${ }^{1}$ Giovanni Paolo II, Codice di Diritto Canonico. Testo ufficiale e versione italiana [CDC 1983], can. $586 \$ 1$, Unione Editori Cattolici Italiani, Roma 1983, 389.

${ }^{2}$ IX SinOdo DeI Vescovi, Instrumenutm laboris, n. 81, in: Documenti sulla vita religiosa (1990-1996), II, raccolti da G.F. Poli e P. Crespi, Editrice Elle Di Ci, Torino 1999, 293. Cfr. A. Montan, Costituzioni. Attenzioni giuridiche, in: http://servidimaria.net/sitoosm/it/testi-osm/documenti-osm/costcanon.pdf, p. 2.
} 
è indipendenza degli istituti rispetto all'autorità gerarchica"3 e "non ha un carattere assoluto, ma (...) è limitata all'esterno dalla potestà della Sede Apostolica o del Vescovo diocesano" ", a seconda che si tratti di un istituto religioso di diritto pontificio o di diritto diocesano ${ }^{5}$. A. Montan osserva che il principio della «giusta autonomia» si concretizza tra l'altro "nel riconoscimento ai singoli istituti religiosi del dovere-diritto di darsi norme proprie ispirate all'identità carismatica specifica, conformi, nella forma e nei contenuti, a quanto stabilito dallo stesso ordinamento (...)"6 della Chiesa. Il Codice di Diritto Canonico, tutelando una giusta autonomia dei singoli istituti religiosi, stabilisce che le norme contenute nello stesso Codice sono obbligatorie per tutti gli istituti religiosi, definisce materie che debbono essere regolate nel codice fondamentale, ossia nelle Costituzioni, e/o materie che debbono essere raccolte in altri codici.

\section{L'articolazione del diritto dei singoli istituti religiosi}

Tenendo conto delle norme del Codice di Diritto Canonico occorre constatare che il diritto di ogni istituto religioso comprende: 1) le norme del diritto universale, emanate dalla Sede Apostolica, contenuta nel Codice di Diritto Canonico e nelle altre leggi canoniche extracodiciali; 2) le norme di diritto particolare, emanate dai Vescovi diocesani e dalle conferenze dei vescovi su materie di loro competenza e vincolanti gli istituti religiosi presenti nei loro territori; 3) il codice

\footnotetext{
${ }^{3}$ IX Sinodo Dei Vescovi, Instrumenutm laboris, n. 81, in: Documenti sulla vita religiosa (1990-1996), II, 293.

${ }^{4}$ A. Montan, Costituzioni. Attenzioni giuridiche, in: http://servidimaria.net/ sitoosm/it/testi-osm/documenti-osm/costcanon.pdf, p. 2.

${ }^{5}$ Il Codice di Diritto Canonico stabilisce: "Fermo restando il disposto del can. 586, gli istituti di diritto pontificio sono soggetti in modo immediato ed esclusivo alla potestà della Sede Apostolica in quanto al regime interno e alla disciplina" (can. 593). "L'istituto di diritto diocesano, fermo restando il can. 586, rimane sotto la speciale cura del Vescovo diocesano" (can. 594).

${ }^{6}$ A. Montan, Costituzioni. Attenzioni giuridiche, in: http://servidimaria.net/ sitoosm/it/testi-osm/documenti-osm/costcanon.pdf, pp. 2-3.
} 
fondamentale, ossia le Costituzioni di ogni istituto religioso; 4) le norme raccolte in altri codici, distinti dalle Costituzioni? ${ }^{7}$.

Le norme contenute nelle Costituzioni e nei codici aggiunti costituiscono insieme diritto proprio di ogni istituto religioso che quindi si distingue dal diritto universale, emanato dalla Sede Apostolica, e dal diritto particolare, emanato dalle Chiese particolari.

\subsection{Le Costituzioni e il loro scopo}

Dalla lettura dei primi tre paragrafi del can. 587 del Codice vigente risulta che ogni istituto religioso deve avere un codice fondamentale o il testo delle costituzioni e che lo scopo principale di tale codice è quello di "custodire più fedelmente la vocazione e l'identità propria dei singoli istituti"s. Il nome usato dal legislatore, ossia il codice fondamentale, è molto indicativo perché sottolinea che si tratta di un complesso delle norme su cui si fonda tutto il resto; esso quindi forma il diritto costitutivo e stabile. Lo stesso legislatore in seguito determina il contenuto delle Costituzioni ${ }^{9}$, i criteri della loro redazione ${ }^{10} \mathrm{e}$ l'autorità competente per la loro approvazione ${ }^{11}$.

\subsubsection{Il contenuto delle Costituzioni}

Dal $₫ 1$ del can. 587 e dal richiamato in esso can. 578 risulta che le Costituzioni di ciascun istituto religioso devono contenere la descrizione del patrimonio dell'istituto in tutti i suoi elementi: la

${ }^{7}$ Sull'articolazione del diritto proprio degli istituti religiosi scrivono tra l'altro: E. GAmbARI, I religiosi nel Codice. Commento ai singoli canoni, Editrice Àncora, Milano 1986, 53-58; A. CAlabrese, Gli istituti religiosi. Lineamenti di diritto canonico, Fonti Vive, Roma 1986, 57-64; J. BEyER, Il diritto della vita consacrata, Editrice Àncora, Milano 1989, 87-97; D. Andrés, Le forme di vita consacrata. Commento teologico-giuridico al Codice di diritto Canonico, Ediurcla, Roma 2014, 54-59 (con abbondante bibliografia alla nota 18, pp. 54-55); V. DE PAOLIs, La vita consacrata nella Chiesa, Marcianum Press, Venezia 2015, 224-231.

${ }^{8}$ CDC 1983, can. $587 \$ 1$.

${ }^{9} \mathrm{Cf}$. ibidem.

${ }^{10}$ Cf. ibidem, can. $587 \$ 3$.

${ }^{11} \mathrm{Cf}$. ibidem, can. $587 \$ 2$. 
natura, il fine, lo spirito e l'indole dell' istituto, secondo l'intendimento e i progetti dei fondatori sanciti dalla competente autorità della Chiesa, nonché le sane tradizioni dell'istituto; poi le norme fondamentali relative al governo dell'istituto, alla disciplina dei membri, alla loro incorporazione e formazione; infine l'oggetto proprio dei vincoli sacri mediante cui si professano i consigli evangelici di castità, di povertà e di obbedienza. Si tratta delle norme basilari che costituiscono il fondamento sul quale si innesteranno le norme degli altri codici. Tali norme fondamentali però devono sempre rispettare le norme del diritto universale della Chiesa, a meno che la Sede Apostolica abbia voluto concedere un privilegio ${ }^{12}$ o una dispensa ${ }^{13}$.

Occorre però notare che il contenuto delle Costituzioni, indicato nel canone $587 \S 1$, non è un indice dettagliato, ma solo una traccia da cui non si può prescindere nel definire le questioni essenziali da includere nelle Costituzioni ${ }^{14}$. Difatti, nel Codice di Diritto Canonico, soprattutto nella parte dedicata alle norme comuni a tutti gli istituti di vita consacrata ${ }^{15}$ ed agli istituti religiosi ${ }^{16}$, si trovano puntuali rinvii - in questioni concrete - alle Costituzioni o al diritto proprio. Con il rinvio alle Costituzioni la Chiesa vuole che la materia - considerata importante - sia trattata obbligatoriamente nelle Costituzioni, invece con il rinvio al diritto proprio si lascia ai singoli istituti religiosi la libertà di inserire la norma richiesta nell'uno o nell'altro dei codici,

\footnotetext{
${ }^{12}$ Cf. ibidem, cann. $4,76 \$ 1$.

${ }^{13}$ Cf. ibidem, can. 85.

${ }^{14}$ Cf. A. Montan, Costituzioni. Attenzioni giuridiche, in: http://servidimaria.net/ sitoosm/it/testi-osm/documenti-osm/costcanon.pdf, p. 6.

${ }^{15}$ Cf. CDC 1983, cann. 573-606.

${ }^{16}$ Cf. ibidem, cann. 607-709.
} 
tenendo però presente il criterio generale dei cann. $578^{17}$ e $587^{18}$ ed anche del can. 576 ${ }^{19}$.

Oltre ai canoni indicati poco fa, ossia 587 e 578 che parlano esplicitamente del contenuto delle Costituzioni, occorre anche tener conto di moltissimi canoni ${ }^{20}$ che trattano delle questioni riguardanti la natura, il fine, lo spirito e l'indole dell'istituto religioso e delle sane tradizioni ${ }^{21}$; la vita dei religiosi, ossia la consacrazione ${ }^{22}$, l'impegno di vita spirituale $^{23}$, la dimensione fraterna della vita consacrata religiosa ${ }^{24}$, il dinamismo apostolico ${ }^{25}$, l'incorporazione e la formazione ${ }^{26}$; il governo dell'istituto religioso ${ }^{27}$.

I rinvii espliciti alle Costituzioni si trovano:

- $\quad$ nelle norme comuni a tutti gli Istituti di vita consacrata: 581 (divisione dell'Istituto in parti e autorità competente a erigere,

17 "L'intendimento e i progetti dei fondatori, sanciti dalla competente autorità della Chiesa, relativamente alla natura, al fine, allo spirito e all'indole dell'istituto, così come le sane tradizioni, cose che costituiscono il patrimonio dell'istituto, devono essere da tutti fedelmente custoditi."

18 "Per custodire più fedelmente la vocazione e l'identità dei singoli istituti il codice fondamentale, o costituzioni, di ciascuno deve contenere, oltre a ciò che è stabilito da osservarsi nel can. 578, le norme fondamentali relative al governo dell'istituto e alla disciplina dei membri, alla loro incorporazione e formazione, e anche l'oggetto proprio dei sacri vincoli."

19 "Spetta alla competente autorità della Chiesa interpretare i consigli evangelici, regolarne la prassi con leggi, costituirne forme stabili di vita mediante l'approvazione canonica e parimenti, per quanto le compete, curare che gli istituti crescano e si sviluppino secondo lo spirito dei fondatori e le sane tradizioni."

${ }^{20}$ Cf. A. Montan, Costituzioni. Attenzioni giuridiche, in: http://servidimaria.net/ sitoosm/it/testi-osm/documenti-osm/costcanon.pdf, pp. 7-9.

${ }^{21}$ Cf. CDC 1983, cann. $588 \$ \$ 1-3,589,603$ ss, 608, 674-675, 677 $\$ 1$.

${ }^{22}$ Cf. ibidem, cann. 573-575, 590, 598-601, $607 \$ 1$.

${ }^{23}$ Cf. ibidem, cann. $663 \$ \$ 1-5,664$.

${ }^{24}$ Cf. ibidem, cann. 602, $607 \$ 2,608,665 \$ 1$.

${ }^{25}$ Cf. ibidem, cann. 673-678, 682.

${ }^{26}$ Cf. ibidem, cann. $597 \$ 2,642,659 \$ 1,660 \$ \$ 1-2,641,643 \$ 1,645-649,650 \$ 2$, $651,652 \$ 2-5,654,656-657,661,670,689,690 \$ 1$.

${ }^{27}$ Cf. ibidem, cann. 618-624, 625, $627 \$ 1,631 \S \$ 1-2,634 \$ 1,968 \S 2,969 \$ 2,1019$ $\S 1,670,684-704$. 
unire e modificare i limiti di queste parti), $596 \$ 1$ (potestà dei superiori e dei capitoli), $598 \$ 1$ (modo di vivere i singoli consigli evangelici in conformità all'indole e alle finalità proprie dell'Istituto), 601 (misura in cui il contenuto del consiglio diviene oggetto del voto di obbedienza) ${ }^{28}$;

- nelle norme riguardanti gli Istituti religiosi: $609 \$ 1$ (autorità competente a erigere una casa), 614 (autorità del superiore di un istituto religioso su un monastero sui iuris), $616 \$ 1$ (soppressione di una casa religiosa eretta legittimamente), 623 (periodo adeguato di tempo di incorporazione all' istituto, dopo la professione perpetua (solenne), per l'elezione o la nomina a superiore maggiore), $624 \$ 1$ (durata e permanenza nell'ufficio di moderatore supremo), $625 \$ \$ 1$ e 3 (elezione canonica del moderatore supremo), $627 \$ 1$ (necessità del consiglio del superiore, composizione e valorizzazione), $631 \$ \$$ 1-2 (autorità del capitolo generale), $634 \$ 1$ (capacità economica dell'Istituto, delle province e delle case), $648 \$ 2$ (periodi di esperienze apostoliche formative), 662 (obbligo della sequela di Cristo quale è espressa nelle Costituzioni dell’istituto), $667 \$ 3$ (clausura adatta all'indole propria e alla missione dell' istituto), $668 \$ 1$ (determinazione circa l'uso e l'usufrutto dei beni, avanti la prima professione), 670 (doveri dell'istituto verso i propri membri) ${ }^{29}$;

- nelle altre parti del Codice: 765 (superiore competente a concedere la licenza per predicare ai religiosi nelle loro case o oratori), 832 (superiore competente a concedere la licenza per pubblicare scritti che trattano questioni di religione o di costumi), 833 n. 8 (professione di fede da emettere

\footnotetext{
${ }^{28}$ Cf. A. Montan, Costituzioni. Attenzioni giuridiche, in: http://servidimaria.net/ sitoosm/it/testi-osm/documenti-osm/costcanon.pdf, p. 9; E. GAMBARI, Il nuovo Codice e la vita religiosa. Studi, Editrice Àncora, Milano1984, 209-210.

${ }^{29}$ Cf. A. Montan, Costituzioni. Attenzioni giuridiche, in: http://servidimaria.net/ sitoosm/it/testi-osm/documenti-osm/costcanon.pdf, p. 10; E. GAMBARI, Il nuovo Codice e la vita religiosa, 210-213.
} 
personalmente dai superiori degli istituti religiosi clericali), $1019 \$ 1$ (facoltà di concedere le lettere dimissorie ai membri ascritti all’istituto), $1174 \$ 1$ (obbligo di celebrare la liturgia delle ore), $1337 \$ 1$ (ingiunzione di dimorare in un determinato luogo o territorio), $1427 \$ \$ 1-2$ (soluzione di controversie tra religiosi o case o province dell'istituto) ${ }^{30}$.

Tutti questi riferimenti costituiscono un aiuto e una guida per l'elaborazione oppure per la revisione o la modifica del diritto proprio, ma in concreto molto dipende dal carattere e dalle tradizioni di ogni istituto religioso. Le Costituzioni dei Carmelitani Scalzi aggiungono: "Le Costituzioni interpretano e spiegano la Regola secondo lo spirito e l'intenzione dei nostri Fondatori e la propongono ai religiosi perché la osservino. Perciò devono essere considerate quali leggi fondamentali dell'Ordine insieme alla Regola"31.

Il motu proprio Ecclesiae Sanctae stabiliva che dalle Costituzioni si doveva escludere "gli elementi già caduti in disuso, o soggetti a mutazioni secondo i costumi di ciascuna epoca, o che rispondono a consuetudini puramente locali"32.

\subsubsection{I criteri della redazione delle Costituzioni}

Le indicazioni sui criteri della redazione delle Costituzioni vengono date dal $\$ 3$ del can. 587: "In tale codice - dice il testo - siano adeguatamente armonizzati gli elementi spirituali e quelli giuridici; tuttavia non si moltiplichino le norme senza necessità". Difatti, la vita consacrata religiosa non può essere descritta sufficientemente solo con

\footnotetext{
${ }^{30}$ Cf. A. Montan, Costituzioni. Attenzioni giuridiche, in: http://servidimaria.net/ sitoosm/it/testi-osm/documenti-osm/costcanon.pdf, p. 11; E. GAMBARI, Il nuovo Codice e la vita religiosa, 213-214.

${ }^{31}$ Cf. Costituzioni dei Fratelli Scalzi dell'Ordine della Beata Vergine Maria del Monte Carmelo [Costituzioni OCD 1986], n. 149, in: Regola. Costituzioni. Norme applicative dei Fratelli Scalzi dell'Ordine della Beata Vergine Maria del Monte Carmelo, II ristampa, Roma 1999, 40.

${ }^{32}$ Paolo VI, Motu proprio Ecclesiae Sanctae [ES], 06.08.1966, art. 14, in: Documenti sulla vita religiosa (1963-1990), I, raccolti da J. Aubry, Editrice Elle Di Ci, Torino 1990, 54.
} 
le norme giuridiche; tale descrizione richiede necessariamente anche la dimensione storica, dottrinale e spirituale. Lo sottolineava già il decreto conciliare Perfectae caritatis $^{33}$ e il motu proprio postconciliare Ecclesiae Sanctae ${ }^{34}$. Se poi questi elementi sono adeguatamente armonizzati, essi s'illuminano a vicenda e si completano. Il legislatore invita inoltre che nella redazione delle Costituzioni si curi la loro completezza e anche la loro brevità limitandosi solo alle norme basilari e rinviando agli altri codici le norme secondarie, anche se necessarie. Infatti, già prima del Concilio Vaticano II il Magistero della Chiesa raccomandava la terminologia precisa, la brevità, la chiarezza, un ottimo ordine ${ }^{35}$, ed il decreto conciliare Perfectae caritatis ricorda che "Tutti però si ricordino che la speranza del rinnovamento è da riporsi in una più diligente osservanza della regola e delle costituzioni che nel moltiplicare le leggi"36.

I criteri indicati occorre tenerli presenti sia nell'elaborazione delle Costituzioni sia nella loro modifica.

\subsection{Altri codici}

Oltre alle Costituzioni, il $\$ 4$ del can. 587 prevede altre norme e altri codici: "Tutte le altre norme - dice il testo - stabilite dall'autorità competente dell'istituto siano opportunamente raccolte in altri codici e potranno essere rivedute e adattate convenientemente secondo le esigenze dei luoghi e dei tempi". Difatti, il Codice di Diritto Canonico, oltre ai casi in cui stabilisce che determinate questioni debbono essere regolate obbligatoriamente nel codice fondamentale, ossia nelle

\footnotetext{
${ }^{33}$ Cf. Concilio Vaticano II, Perfectae caritatis [PC], 28.10.1965, nn. 2-3, in: Documenti sulla vita religiosa (1963-1990), I, raccolti da J. Aubry, Editrice Elle Di Ci, Torino 1990, 27-29.

${ }^{34}$ Cf. ES art. 13.

${ }^{35}$ Cf. SaCra Congregatio de Religiosis, Normae secundum quas Sacra Congregatio de Religiosis in novis religiosis congregationibus approbandis procedere solet, 06.03.1921, n. 24, in: Leges Ecclesiae post Codicem iuris canonici editae, I, collegit, digessit notisque ornavit X. Ochoa, Commentarium pro Religiosis, Roma 1966, col. 377.
}

${ }^{36}$ PC n. 4. 
Costituzioni, indica anche altre questioni che dovrebbero essere regolate nel diritto proprio, lasciando ai singoli istituti religiosi la libertà di inserire tali norme o nelle Costituzioni o negli altri codici ${ }^{37}$.

Le norme inserite negli altri codici, più facilmente adattabili, sempre conformi al diritto universale della Chiesa e al codice fondamentale, hanno lo scopo di completare o applicare le prescrizioni del diritto universale della Chiesa e delle Costituzioni, regolare le materie e settori in essi non contemplati oppure ordinati nelle Costituzioni solo su punti essenziali. Tali norme possono portare vari nomi (Codici complementari, Norme applicative, Statuti, Regole pratiche, Direttori, Ordinamenti, Regolamenti, Ratio institutionis ossia l'ordinamento della formazione, Norme pratiche dei capitoli, Libri di usanze, Ordinazioni, Determinazioni ecc.). Esse possono riguardare tutto l'istituto religioso o una circoscrizione, o una regione, o una casa religiosa. Il motu proprio Ecclesiae Sanctae ${ }^{38}$ ordinava che codici complementari dovrebbero raccogliere "le norme che corrispondono all'epoca attuale, alle condizioni fisiche, psicologiche dei membri, e a circostanze particolari”.

Le Costituzioni dei Carmelitani Scalzi nel capitolo secondo della terza parte, trattando degli altri codici, parlano delle Norme applicative ${ }^{39}$ e delle Ordinazioni del Capitolo generale ${ }^{40}$.

Le Norme applicative hanno lo scopo di dichiarare e completare le norme delle Costituzioni e mantengono il carattere generale per lasciare spazio a un sano pluralismo in ciò che riguarda le esigenze locali o regionali.

Il diritto proprio carmelitano-teresiano menziona anche altre norme complementari: Norme per la celebrazione del Capitolo Generale

\footnotetext{
${ }^{37}$ Cf. A. Montan, Costituzioni. Attenzioni giuridiche, in: http://servidimaria. net/sitoosm/it/testi-osm/documenti-osm/costcanon.pdf, pp. 11-12; E. GAMBARI, Il nuovo Codice e la vita religiosa, 215-223.

${ }^{38}$ Cf. ES, art. 14.

${ }^{39}$ Cf. Costituzioni OCD 1986, nn. 151 e 153.

${ }^{40}$ Cf. ibidem, nn. 152-153.
} 
ordinario $^{41}$; Ordinazioni del Capitolo provinciale ${ }^{42}$; Ordinazioni del Definitorio per l'amministrazione dei periodici e delle associazioni che si trovano nell'ambito dei nostri conventi o delle nostre chiese ${ }^{43}$; Norme particolari del Consiglio provinciale per l'amministrazione dei periodici e delle associazioni ${ }^{44}$; Determinazioni del Capitolo provinciale $^{45}$; Determinazioni del Definitorio ${ }^{46}$; Determinazioni del Capitolo locale ${ }^{47}$; Istruzione pratica del Definitorio sul modo di procedere nel Capitolo provinciale ${ }^{48}$.

Nell'Ordine dei Carmelitani Scalzi ci sono inoltre i codici che non sono menzionati esplicitamente nelle prescrizioni delle Costituzioni o delle Norme applicative: Ratio institutionis ${ }^{49}$, Istruzione per gli eremi $O C D^{50}$, Istruzione per le parrocchie $O C D^{51}$.

$\mathrm{Ci}$ potrebbero essere anche altre norme complementari: un'istruzione sull'amministrazione dei beni al livello di governo generale, provinciale e locale; un'istruzione sul modo di procedere dei Superiori provinciali e dei Consigli provinciali; un Vademecum di diversi procedimenti amministrativi a livello di governo provinciale e locale; Norme per la visita canonica; Istruzione sul modo di procedere nel capitolo locale; Statuti delle missioni; Rituale o cerimoniale.

\footnotetext{
${ }^{41}$ Cf. ibidem, n. 170 i.

${ }^{42}$ Cf. ibidem, nn. 195 a, 196.

${ }^{43}$ Cf. Norme applicative delle Costituzioni dei Fratelli Scalzi dell'Ordine della Beata Vergine Maria del Monte Carmelo [Norme applicative OCD 1986], n. 258 e, in: Regola. Costituzioni. Norme applicative dei Fratelli Scalzi dell'Ordine della Beata Vergine Maria del Monte Carmelo, II ristampa, Roma 1999, 97.

${ }^{44}$ Cf. ibidem.

${ }^{45}$ Cf. Costituzioni OCD 1986, n. 120; Norme applicative OCD 1986, n. 230.

${ }^{46}$ Cf. Costituzioni OCD 1986, n. 187.

${ }^{47}$ Cf. Norme applicative OCD 1986, n. 27.

${ }^{48}$ Cf. ibidem, n. 217.

${ }^{49}$ Cf. La formazione nel Carmelo Teresiano. Ratio institutionis O.C.D., Casa Generalizia OCD, Roma 1992.

${ }^{50}$ Cf. Istruzione per gli eremi O.C.D., Casa Generalizia OCD, Roma 1993.

${ }^{51}$ Cf. Conferencia IBerICA OCD, Instrucción para las parroquias OCD, Madrid 2003.
} 


\section{L'autorità competente per l'approvazione e la modifica del diritto proprio}

Soffermandosi sull'autorità competente per l'approvazione del diritto proprio e per la sua modifica, occorre distinguere le Costituzioni e gli altri codici.

\subsection{Le Costituzioni}

Il $\$ 2$ del can. 587 stabilisce che le Costituzioni devono essere approvate dalla competente autorità della Chiesa. Nel caso di un istituto religioso di diritto pontificio tale competenza appartiene alla Congregazione per gli Istituti di Vita Consacrata e le Società di Vita Apostolica e deriva dal legame di questi istituti con la Chiesa ${ }^{52}$ e dal compito proprio della Chiesa ${ }^{53}$.

Difatti, ogni istituto religioso può liberamente valutare l'opportunità di modificare qualche norma delle proprie Costituzioni. Normalmente le stesse Costituzioni prevedono la procedura per tale modifica. La prassi vuole che la modifica debba essere proposta con una maggioranza qualificata, in genere di due terzi dei voti. Le Costituzioni dei Carmelitani Scalzi, richiamandosi al can. $587 \$ 2$, affermano che solo la Sede Apostolica può abrogare (la revoca totale), derogare (la revoca parziale) o mutare le Costituzioni $i^{54}$, e stabiliscono che ciò può avvenire "dopo che la cosa sia stata decisa con i due terzi dei voti da due Capitoli generali consecutivi”"55. Siccome non viene precisato che si tratta di due Capitoli generali consecutivi ordinari, significa che possano essere due Capitoli generali consecutivi ordinari o un Capitolo generale ordinario e un consecutivo straordinario oppure due Capitoli generali consecutivi straordinari.

\footnotetext{
${ }^{52}$ Cf. CDC 1983, cann. $573 \$ 2,574,575$.

${ }^{53} \mathrm{Cf}$. ibidem, can. 576. Attualmente solo pochi ordini hanno costituzioni non approvate formalmente dalla Sede Apostolica: Domenicani, Gesuiti, Premonstratensi, Carmelitani. Cf. E. GAmbari, Vita religiosa secondo il Concilio e il nuovo Diritto Canonico, Edizioni Monfortane 1985, nota n. 25, p. 75.

${ }^{54}$ Cf. Costituzioni OCD 1986, n. 150.

${ }^{55}$ Ibidem.
} 
Alla Sede Apostolica è riservata anche l'interpretazione autentica delle Costituzioni $\mathrm{OCD}^{56}$. Invece la risoluzione, con una dichiarazione pratica, di eventuali dubbi sorti su qualche sua norma spetta al Definitorio $^{57}$ e la dispensa dalle sue norme disciplinari è di competenza, a seconda dei casi, del Preposito Generale ${ }^{58}$, del Definitorio ${ }^{59}$ o del Consiglio provinciale ${ }^{60}$.

Occorre anche ricordare che nel caso in cui un istituto religioso di diritto pontificio decide di inserire nelle Costituzioni una determinata questione di cui il Codice di Diritto Canonico dice che debba essere regolata nel diritto proprio, l'approvazione e la modifica di tale norma appartengono alla competenza della Sede Apostolica ${ }^{61}$.

\subsection{Altri codici}

Tutti gli altri codici sono approvati dall'autorità interna d'ogni istituti religioso: Capitoli, Superiori, Consigli ai vari livelli, a seconda della loro competenza. Frequentemente e opportunamente alcuni di questi codici hanno bisogno della conferma dell'autorità superiore, p.es. del Definitorio. Tenendo conto della loro funzione, essi possono essere riveduti e adattati convenientemente dall'autorità che li ha approvati per mantenerli rispondenti alle esigenze dei luoghi, dei tempi, dei cambiamenti nella Chiesa e nella società. Alla stessa autorità spetta la loro interpretazione autentica. All'autorità interna compete anche la dispensa da esse.

Nell'Ordine dei Carmelitani Scalzi fanno parte degli altri codici, tra l'altro, le Norme applicative e le Ordinazioni del Capitolo Generale.

Le Norme applicative entrano in vigore dopo l'approvazione del Capitolo generale e possono essere modificate dai successivi Capitoli generali $^{62}$. Per la loro modifica o abrogazione definitiva si richiede

\footnotetext{
${ }^{56}$ Cf. ibidem.

${ }^{57}$ Cf. ibidem.

${ }^{58}$ Cf. ibidem, n. 176.

${ }^{59}$ Cf. Norme applicative OCD 1986, n. 197 i-j.

${ }^{60}$ Cf. ibidem, n. 226 b.

${ }^{61}$ Cf. CDC 1983, can. $587 \$ 2$.

${ }^{62}$ Cf. Costituzioni OCD 1986, n. 151.
} 
l'approvazione con la maggioranza dei due terzi dei voti di due Capitoli generali consecutivi ${ }^{63}$.

Le Ordinazioni sono le norme stabilite dal Capitolo generale con la maggioranza dei due terzi dei voti e obbligano tutto l'Ordine; esse decadono se nel Capitolo generale ordinario immediatamente successivo non sono approvate con la stessa maggioranza di voti ${ }^{64}$.

\section{Il ruolo delle Costituzioni nel rinnovamento della vita religiosa ed il loro aggiornamento}

Il Magistero postconciliare della Chiesa fa appello ai religiosi di rinnovare continuamente la loro vita e la loro missione nella Chiesa invitandoli alla fedeltà creativa. In questo processo di rinnovamento un ruolo importante adempiono proprio le Costituzioni dei singoli istituti religiosi. Ne parlano, tra l'altro, due documenti della Chiesa, ossia l'esortazione apostolica post-sinodale Vita consecrata di san Giovanni Paolo II $^{65}$ e l'istruzione Ripartire da Cristo sul rinnovato impegno della vita consacrata nel terzo millennio della Congregazione per gli Istituti di Vita Consacrata e le Società di Vita Apostolica ${ }^{66}$.

San Giovanni Paolo II spiega che la fedeltà creativa dei singoli religiosi, delle loro comunità e degli istituti interi consiste:

- nella fedeltà all'intraprendenza, all'inventiva ed alla santità dei fondatori come risposta ai segni dei tempi emergenti nel mondo di oggi;

- nella perseveranza nel cammino di santità attraverso le difficoltà materiali e spirituali che segnano le vicende quotidiane;

\footnotetext{
${ }^{63}$ Cf. ibidem, nn. 153 e 152.

${ }^{64}$ Cf. ibidem, n. 152.

${ }^{65}$ Giovanni Paolo II, Esortazione apostolica post-sinodale Vita consecrata [VC], 25.03.1996, in: Documenti sulla vita religiosa (1990-1996), II, raccolti da G.F. Poli e P. Crespi, Editrice Elle Di Ci, Torino 1999, 347-471.

${ }^{66}$ Congregazione per gli Istituti di Vita Consacrata e le Società di Vita Apostolica, Ripartire da Cristo: un rinnovato impegno della vita consacrata nel terzo millennio. Istruzione [RdC], 19.05.2002, in: Enchiridion Vaticanum, vol. 21, a cura di E. Lora, Edizioni Dehoniane Bologna 2005, 311-367.
} 
- nella ricerca della competenza nel proprio lavoro;

- nella coltivazione di una fedeltà dinamica alla missione propria dell'Ordine, adattandone le forme, quando è necessario, alle nuove situazioni e ai diversi bisogni, in piena docilità all'ispirazione divina e al discernimento ecclesiale ${ }^{67}$.

Tale fedeltà è facilitata quando si ricerca sinceramente la conformazione sempre più piena al Signore e quando esiste un rinnovato riferimento alla Regola e alle Costituzioni, perché in essi è racchiuso un itinerario di sequela di Cristo qualificato da uno specifico carisma autenticato dalla Chiesa ${ }^{68}$. Un'accresciuta considerazione per la Regola e per le Costituzioni non mancherà di offrire ai religiosi "un criterio sicuro per ricercare le forme adeguate di una testimonianza che sappia rispondere alle esigenze del momento senza allontanarsi dall'ispirazione iniziale"69.

L'istruzione Ripartire da Cristo, parlando del rinnovamento costante della vita e della missione degli istituti religiosi, sottolinea la necessità del rinnovamento continuo della vita spirituale dei religiosi e della spiritualità dell'istituto religioso che si realizza mediante il ritrovamento delle proprie radici della chiamata divina, mediante il confronto con le fonti del carisma proprio dell'istituto e dei suoi testi costituzionali, sempre aperti a nuove e più impegnative interpretazioni, mediante l'approfondimento - nell'attuale stagione della Chiesa - di una spiritualità più ecclesiale e comunitaria, più esigente e matura nel reciproco aiuto verso il raggiungimento della santità, più generosa nelle scelte apostoliche, più aperta a diventare pedagogia e pastorale della santità all'interno dell' istituto religioso e nella sua irradiazione a favore di tutto il popolo di Dio ${ }^{70}$. L'istruzione sottolinea anche il ruolo della Parola di Dio nel rinnovamento della vita spirituale dei religiosi perché l'itinerario della sequela di Cristo, qualificato dal carisma proprio dell'istituto, racchiuso nella Regola

\footnotetext{
${ }^{67}$ Cf. VC 37.

${ }^{68}$ Cf. ibidem.

${ }^{69}$ Ibidem.

${ }^{70}$ Cr. RdC 20.
} 
e nelle Costituzioni, e approvato dalla Chiesa, traduce la particolare interpretazione del Vangelo data dai Fondatori, docili all'impulso dello Spirito Santo, ed aiuta tutti i religiosi a vivere concretamente secondo la Parola di Dio ed essere servi della Parola di Dio nell'impegno della nuova evangelizzazione che costituisce la priorità per la Chiesa nel terzo millennio ${ }^{71}$.

Difatti, le Costituzioni, elaborate e rinnovate secondo le direttive del Magistero della Chiesa, contengono delle norme che scaturiscono da una solida base biblico-teologica, da una comprensione approfondita del carisma fondazionale e della storia dell'istituto religioso, dalla lettura evangelica dei segni dei luoghi e dei tempi, dai cambiamenti che avvengono nella Chiesa e nella società. Tutto ciò comporta a sua volta che le Costituzioni, ed in genere il diritto proprio, non costituiranno una legge scritta in modo fisso e immutabile, ma una legge al servizio della vita e quindi aperta a possibili approfondimenti dottrinali e carismatici, adattamenti e aggiornamenti. Le Costituzioni dovrebbero essere viste "come un libro di vita nel quale sono contenuti gli atteggiamenti che i religiosi devono tradurre nella loro vita e nel loro operato ogni giorno"72, "una sicura guida alla santità"73, "un aiuto specifico per vivere la vocazione ricevuta da Dio"74. Il Magistero della Chiesa ed anche la riflessione teologica sulla vita religiosa ritengono che per una fedeltà creativa ed un rinnovamento fedele all'ispirazione originaria occorrono un rinnovato riferimento alle Costituzioni, la fedeltà alle Costituzioni, la promozione - nell'ambito della formazione permanente - di una lettura continua e uno studio approfondito delle Costituzioni, fatti alla luce dei segni dei luoghi e

\footnotetext{
${ }^{71}$ Cf. ibidem, 24.

${ }^{72}$ Per una fedeltà perseverante. L'attualità delle costituzioni nella vita degli istituti religiosi, 11.04.2010, in: http://www.osservatoreromano.va/it/news/ per-una-fedelta-perseverante.

${ }^{73}$ Ibidem.

${ }^{74}$ Ibidem. Sul ruolo delle Costituzioni nella vita religiosa cfr. J. Alvarez Gómez, Las Constituciones, libro de vida, Instituto Teológico de Vid Religiosa, Madrid 1987; M. Díez Presa, Las Constituciones. Lectura teológica y oracional, Publicaciones Claretianas, Madrid 1998.
} 
dei tempi, non escludendo la possibilità di un loro aggiornamento ${ }^{75}$. Tale aggiornamento delle Costituzioni e degli altri codici deve però basarsi sempre "su una retta e adeguata comprensione del carisma di fondazione" "76, "sulle esigenze proprie dei luoghi e dei tempi"77 e deve farsi secondo le esigenze della Chiesa e della società in cui gli istituti religiosi e le loro comunità vivono e svolgono il loro servizio apostolico $^{78}$.

L'aggiornamento del diritto proprio, e quindi anche delle Costituzioni, può avvenire su iniziativa dello stesso istituto religioso come espressione della sua fedeltà creativa oppure su iniziativa del Magistero della Chiesa. Quest'ultima situazione si è verificata recentemente ad opera della Congregazione per gli Istituti di Vita Consacrata e le Società di Vita Apostolica.

Difatti, la Congregazione, nella Lettera circolare Linee orientative per la gestione dei beni, del 2 agosto $2014^{79}$, ha chiesto agli istituti di vita consacrata di stabilire nel diritto proprio le norme riguardanti alcune questioni particolari relative all'amministrazione dei beni: l'obbligatorietà dell'introduzione nelle Costituzioni e/o in un altro testo del diritto proprio del concetto di patrimonio stabile $e^{80}$, la legittima assegnazione di un bene al patrimonio stabile ${ }^{81}$.

\footnotetext{
${ }^{75}$ Cf. Per una fedeltà perseverante. L'attualità delle costituzioni nella vita degli istituti religiosi, 11.04.2010, in: http://www.osservatoreromano.va/it/news/ per-una-fedelta-perseverante.

${ }^{76}$ Ibidem.

${ }^{77}$ Ibidem.

${ }^{78}$ Cf. Y. Sugawara, Il ruolo delle costituzioni negli istituti di vita consacrata (can. 587), Periodica 98/4 (2009) 687.

${ }^{79}$ Congregazione per gli Istituti di Vita Consacrata E le Società di Vita Apostolica, Lettera circolare Linee orientative per la gestione dei beni negli istituti di vita consacrata e le Società di vita apostolica, 02.08.2014, Libreria Editrice Vaticana, Città del Vaticano 2014.

${ }^{80}$ Cf. ibidem, 16.

${ }^{81}$ Cf. ibidem, 15.
} 
La stessa Congregazione nel documento Per vino nuovo otri nuovi. Orientamenti, del 6 gennaio 201782, chiede anche la revisione delle norme del diritto proprio riguardanti alcune questioni (il cap. III: Preparare otri nuovi): "una modifica delle strutture, in modo che per tutti siano di sostegno in una rinnovata fiducia che rilanci una fedeltà dinamica e fraterna"83, una revisione della propria Ratio formationis ${ }^{84}$, una migliore recezione della norma del Codice di Diritto Canonico contenuta nel can. $624 \$ 2^{85}$, la composizione dei capitoli e dei consigli generali e provinciali ${ }^{86}$.

Un terzo documento della stessa Congregazione, ossia Economia a servizio del carisma e della missione. Orientamenti, del 6 gennaio $2918^{87}$, richiede obbligatoriamente o raccomanda, a seconda dei casi, di stabilire nel diritto proprio norme riguardanti vari aspetti particolari dell'amministrazione dei beni e della gestione delle opere ${ }^{88}$.

Riferendosi direttamente alle Costituzioni OCD, occorre sottolineare che esse, rinnovate secondo le direttive conciliari e postconciliari e approvate dalla Chiesa, sono veramente espressione e guida della spiritualità dell'Ordine e per questo dovrebbero essere oggetto di una lettura assidua fatta dalla prospettiva della propria cultura, dovrebbero essere studiate con diligenza, perché tracciano un cammino per una fedele sequela di Gesù Cristo secondo il carisma carmelitano-teresiano donato da Dio alla Chiesa e approvato dal Magistero della Chiesa.

\footnotetext{
${ }^{82}$ Congregazione per gli Istituti di Vita Consacrata e le Società di Vita Apostolica, Per vino nuovo otri nuovi. Dal Concilio Vaticano II la vita consacrata e le sfide ancora aperte. Orientamenti, 06.01.2017, Libreria Editrice Vaticana, Città del Vaticano 2017.

${ }^{83}$ Cf. ibidem, n. 33.

${ }^{84}$ Cf. ibidem, nn. 34-37.

${ }^{85}$ Cf. ibidem, n. 46 - la rielezione dei superiori.

${ }^{86}$ Cf. ibidem, nn. 48-54.

${ }^{87}$ Congregazione per gli Istituti di Vita Consacrata e le Società di Vita Apostolica, Economia a servizio del carisma e della missione. Orientamenti, 06.01.2018, Libreria Editrice Vaticana, Città del Vaticano 2018.

${ }^{88}$ Cf. ibidem, nn. 58, 60, 61, 63-64, 67, 70, 71-72, 75, 80, 87, 89.
} 


\section{Il percorso dell'elaborazione del diritto proprio $\mathrm{OCD}$ vigente}

L'aggiornamento del diritto proprio dell'Ordine dei Carmelitani Scalzi richiede molteplici competenze (tra cui quelle teologiche, storiche, carismatiche, giuridiche, culturali) ed un adeguato procedimento, assieme al tempo necessario, a seconda dell'ampiezza e profondità della revisione dei testi normativi (rielaborazione, semplice revisione, adattamenti necessari). Conviene perciò conoscere, almeno genericamente, il modo di procedere nell'elaborazione delle norme del diritto proprio, adoperato dopo il Concilio Vaticano II, per farsi un'idea sulle esigenze e conseguenze della decisione di aggiornarlo nel momento presente della storia dell'Ordine, della Chiesa e della società. Da questa procedura si possono scegliere degli elementi - a seconda dell'intensità dell'aggiornamento - che garantiscano un lavoro serio, responsabile, utile ed inducano a impegnarsi, a livello personale e comunitario, nell'approfondimento dell'identità vocazionale dei carmelitani scalzi e della loro missione nella Chiesa al tempo di oggi.

La storia della revisione e redazione delle Costituzioni OCD vigenti, ed in genere del diritto proprio, è iniziata durante il Capitolo Generale Speciale del 1967 e del 1968 ed è terminata nell'anno 1986 quando la Sede Apostolica ha approvato il testo definitivo adattato al Codice di Diritto Canonico del $1983^{89}$. Quindi tutto il processo legislativo durò 20 anni ed in esso fu impegnato tutto l'Ordine: il Definitorio Generale, i Capitoli Generali Ordinari e Straordinari, le diverse Commissioni, i Definitori Straordinari, le Province con i loro Capitoli Provinciali e con le loro comunità locali.

Nel percorso del processo della rielaborazione del diritto proprio dei Carmelitani Scalzi possiamo individuare i seguenti momenti:

- l'istruzione del Definitorio riguardante il tema del rinnovamento adeguato della vita religiosa e l'organizzazione del lavoro di riflessione con la partecipazione delle comunità

${ }^{89}$ Cf. Congregatio pro Religiosis et Institutis Saecularibus, Novum decretum, 05.03.1986, in: Acta Ordinis Carmelitarum Discalceatorum [Acta OCD] 31 (1986) 9. 
e dei Capitoli provinciali del 1966 alla revisione del diritto proprio dell'Ordine ${ }^{90}$;

- il Capitolo Generale Speciale del 1967 (V-VI) - 1968 (IX-X) con il lavoro delle sette Commissioni Capitolari e la preparazione dei sette Decreti Capitolari (la vita e lo spirito dell'Ordine, i fratelli non chierici, l'apostolato, la formazione, il governo, l'amministrazione dei beni, gli elementi obsoleti e le esperienze) e la susseguente promulgazione dei sette Decreti (il Preposito Generale, 31.12.1968) ${ }^{91}$;

- la sperimentazione, la riflessione e la valutazione dei Decreti (la dottrina e le norme) da parte delle comunità e dei Capitoli provinciali (le osservazioni) (1968-1973) ${ }^{92}$;

- il Capitolo Generale del 1973, chiamato Capitolo di "sincerità", e l'elaborazione delle Normae OCD - $1973^{93}$;

- la Commissione post-capitolare, nominata dal Definitorio, per la redazione delle Costituzioni e del Direttorio, secondo

\footnotetext{
${ }^{90} \mathrm{Cf}$. Definitorium Generale, Instructio de accommodata renovatione vitae nostrae ad mentem Concilii Oecumenici Vaticani II ordinanda, 01.07.1965, in: Acta OCD 10 (1965) 120-128.

${ }^{91}$ Cf. Capitulum Generale LXXXIII, 14.05-06.07.1967, in: Acta OCD 12 (1967) 10-65; Praepositus Gener alis OCD, Decretum, 31.12.1968, in: Decreta Capituli Specialis O.C.D. 1968, VII. Cf. anche P.R. Zubieta, El derecho de los Carmelitas Descalzos. Comentario doctrinal y jurídico a las Constituciones de la Orden de Carmelitas Descalzos (1986), Editorial Monte Carmelo, Burgos 2008, 17-18.

${ }^{92}$ Cf. Definitorium Extraordinarium, De Commissione ad Schema nostrarum Legum apparandum, 21.09.1972, in: Acta OCD 16-18 (1971-1973) 210-211; Definitorium Generale, Convocatio Commissionis ad Schema Legum apparandum, 22.09.1972, in: Acta OCD 16-18 (1971-1973) 137-138; IDEM, Schema Legum nostrarum in Provincis mittatur, 08.12.1972, in: Acta OCD 16-18 (1971-1973) 138. Cf. anche P.R. Zubieta, El derecho de los Carmelitas Descalzos, 19.

${ }^{93}$ Cf. Capitulum Generale LXXXIV, 10.06-10.07.1973, in: Acta OCD 18-19 (1973-1974) 53-95; Normae Capituli Generalis O.C.D. - 1973, Curia Generalis O.C.D., Romae 1973. Cf. anche P.R. Zubieta, El derecho de los Carmelitas Descalzos, 19-20.
} 
i criteri indicati dal Capitolo Generale del 1973 e sotto la cura del Definitorio (XI 1973 - I 1974) ${ }^{94}$;

- l'esame dei testi delle Costituzioni e del Direttorio da parte degli esperti nelle Province e le loro osservazioni (I 1974 - VIII 1974) $)^{95}$;

- il lavoro della Commissione post-capitolare: l'esame delle osservazioni e proposte degli esperti delle Province ed il perfezionamento dei testi (IX 1974) ${ }^{96}$;

- l'esame dei testi perfezionati da parte delle comunità e dei Capitoli provinciali del 1975 (X 1974 - VI 1975) ${ }^{97}$;

- il lavoro della Commissione post-capitolare: l'esame delle osservazioni e delle proposte dei Capitoli provinciali e la correzione dei testi (X-XII 1975) ${ }^{98}$;

- il lavoro del Definitorio: l'esame ed il perfezionamento dei testi (I-II 1976) e la loro approvazione (03.05.1976) ${ }^{99}$;

- il Definitorio Straordinario del 1976: la presentazione ai partecipanti e l'approvazione "ad experimentum" delle

\footnotetext{
${ }^{94}$ Cf. Definitorium Gener ale, Commissio post-capitularis a Capitulo Generali statuta ad redigendos textus Constitutionum et Directorii O.N., 05.10.1973, in: Acta OCD 18-19 (1973-1974) 96-98. Cf. anche P.R. ZubietA, El derecho de los Carmelitas Descalzos, 20.

${ }^{95}$ Cf. Definitorium Generale, Commissio post-capitularis a Capitulo Generali statuta ad redigendos textus Constitutionum et Directorii O.N., 05.10.1973, in: Acta OCD 18-19 (1973-1974) 99. Cf. anche P.R. Zubieta, El derecho de los Carmelitas Descalzos, 20.

${ }^{96}$ Cf. Definitorium Generale, Commissio post-capitularis a Capitulo Generali statuta ad redigendos textus Constitutionum et Directorii O.N., 05.10.1973, in: Acta OCD 18-19 (1973-1974) 99. Cf. anche P.R. Zubieta, El derecho de los Carmelitas Descalzos, 20.

${ }^{97}$ Cf. Definitorium Generale, Commissio post-capitularis a Capitulo Generali statuta ad redigendos textus Constitutionum et Directorii O.N., 05.10.1973, in: Acta OCD 18-19 (1973-1974) 99. Cf. anche P.R. ZubietA, El derecho de los Carmelitas Descalzos, 20.

${ }^{98}$ Cf. ibidem.

${ }^{99}$ Cf. ibidem, 20-21.
} 
Costituzioni e del Direttorio (dal 16.07.1976 al Capitolo Generale del 1979) ${ }^{100}$;

- le ulteriori osservazioni e proposte dei Capitoli provinciali del 1978 riguardanti le Costituzioni ad experimentum del $1976^{101}$;

- il lavoro della Commissione pre-capitolare: l'elaborazione della "Sinopsis" delle osservazioni e proposte dei Capitoli provinciali del 1978 ${ }^{102}$;

- Il Capitolo Generale del 1979: la revisione sistematica del testo "ad experimentum" tenendo conto dell'apporto delle Province ${ }^{103}$;

- Il perfezionamento del linguaggio del diritto proprio dell'Ordine (le Costituzioni e le Norme applicative), sotto la direzione del Definitorio Generale, terminato durante il Definitorio Straordinario del 1981 (Avon) ${ }^{104}$;

- l'approvazione delle Costituzioni dalla Sede Apostolica il 7 giugno $1981^{105}$;

${ }^{100}$ Cf. Praepositus Generalis, Litterae quibus Definitorium Generale omnes Superiores Provinciales convocat in Congressum, a die 29 maii ad diem 6 iunii 1976, penes Coenobium Sancti Montis Carmeli habendum, 12.02.1976, in: Acta OCD 20/21 (1975-1976) 61-63; IDEM, Allocutio P.N. Praepositi Generalis occasione promulgationis Constitutionum et Directorii Ordinis nostri, 06.06.1976, in: Acta OCD 20/21 (1975-1976) 68-78. Cf. anche P.R. ZubietA, El derecho de los Carmelitas Descalzos, 21.

${ }^{101}$ Cf. Praepositus Generalis, Litterae circulares de Capitulorum Provincialium munere circa novos Constitutionum et Directorii textus iudicium ferendi, 17.09.1977, in: Acta OCD 22-23 (1977-1978) 39-44. Cf. anche P.R. Zubieta, El derecho de los Carmelitas Descalzos, 21.

${ }^{102}$ Cf. ibidem.

${ }^{103}$ Cf. Capitulum Generale LXXXV, 24.04-03.06.1979, in: Acta OCD 24 (1979) 135. Cf. anche P.R. Zubieta, El derecho de los Carmelitas Descalzos, 21.

${ }^{104}$ Cf. Capitulum Generale LXXXV, 24.04-03.06.1979, in: Acta OCD 24 (1979) 135--137; Definitorium Extraordinarium Avonense, 15-26.04.1980, Commissionis Centralis pro studio Legum compositio, in: Acta OCD 25 (1980) 84. Cf. anche P.R. Zubieta, El derecho de los Carmelitas Descalzos, 22.

${ }^{105}$ Cf. Sacra Congregatio pro Religiosis et Institutis Saecularibus, Decretum, 07.06. 1981, in: Acta OCD 26/1 (1981) VII-VIII. 
- l'adattamento delle Costituzioni e delle Norme applicative al nuovo Codice di Diritto Canonico del 1983. Ai lavori parteciparono: il Definitorio Straordinario del 1984 (IX, Villa de Leyva, Colombia), Capitolari del Capitolo Generale del 1985 (osservazioni), la Commissione pre-capitolare (l'elaborazione dell'Instrumentum laboris alla base delle risposte dei Capitolari) ${ }^{106}$;

- il Capitolo Generale del 1985: l'esame e approvazione dei cambiamenti dei testi in forma di proposte (successivamente introdotte da una Commissione, sotto la direzione del Definitorio, nel testo delle Costituzioni e delle Norme applicative ${ }^{107}$;

- l'approvazione definitiva delle Costituzioni dalla Sede Apostolica il 5 marzo $1986^{108}$.

La tappa più importante fu quella del Capitolo Generale Speciale del 1967-1968 con il suo profondo studio sul carisma dell'Ordine dei Carmelitani Scalzi e quindi l'elaborazione dei sette Decreti Capitolari che costituirono la base dottrinale e spirituale per le Costituzioni vigenti. L'Ordine aveva valutato molto positivamente il contenuto dei Decreti menzionati. Lo si vede, tra l'altro, nel messaggio del Capitolo Generale del 1973 in cui leggiamo che i Decreti Capitolari hanno un contenuto dottrinale eccellente e che possono essere definiti come

\footnotetext{
${ }^{106}$ Cf. Definitorium, Commissio pro Legibus nostris aptandis, 18.12.1984, in: Acta OCD 29 (1984) 62; Definitorium Extraordinarium in Villa de Leyva in Columbia diebus 17 ad 25 septembris 1984 celebratum, in: Acta OCD 29 (1984) 68. Cf. anche P.R. Zubieta, El derecho de los Carmelitas Descalzos, 22-23.

${ }^{107}$ Cf. Definitorium Extraordinarium in Villa de Leyva in Columbia diebus 17 ad 25 septembris 1984 celebratum, in: Acta OCD 29 (1984) 73, 96-98; Relatio a P. Pietro Zubieta, Secretario Generali habita de labore post Capitulum Generale peracto quoad textus Constitutionum et Normarum applicativarum Codicis Iuris Canonici aptados, in: Acta OCD 31/II (1986) 113-129. Cf. anche P.R. ZubIETA, El derecho de los Carmelitas Descalzos, 23.

${ }^{108}$ Cf. Congregatio pro Religiosis et Institutis Saecularibus, Novum decretum, 05.03.1986, in: Acta OCD 31 (1986) 9.
} 
riscoperta storica della vocazione religiosa dei carmelitani scalzi nella Chiesa ${ }^{109}$.

\section{Conclusione}

Dalla procedura legislativa, adoperata dall'Ordine negli anni 1968-1986, si possono scegliere degli elementi che siano proficui per la revisione e aggiornamento delle norme del diritto proprio dei Carmelitani Scalzi al tempo di oggi. La ricerca e il discernimento, richiesti da tale lavoro, dovrebbero - a seconda dei casi - incoraggiare o rafforzare o trasformarsi in una lettura teologica e meditativa delle Costituzioni per approfondire la propria identità vocazionale nella Chiesa e - come dice il testo della formula carmelitano-teresiana per la professione religiosa - per vivere fedelmente in unione con la Vergine Maria nell'ossequio di Gesù Cristo, per donarsi con tutto il cuore alla famiglia religiosa iniziata dai Fondatori, per dedicarsi alla perenne orazione e al servizio apostolico della Chiesa, per raggiungere la carità perfetta e per glorificare in eterno la Trinità santissima.

Una accresciuta considerazione per la Regola e per le Costituzioni come ci ricorda san Giovanni Polo II - offrirà ai religiosi "un criterio sicuro per ricercare le forme adeguate di una testimonianza che sappia rispondere alle esigenze del momento senza allontanarsi dall'ispirazione iniziale" 110 , li aiuterà - a livello personale, comunitario ed istituzionale - nel rinnovamento continuo della loro vita religiosa e nel rilanciare una fedeltà dinamica, creativa e fraterna.

\section{The revision of Proper Law of the Teresian Carmel (OCD) in present times}

With regard to the rightful autonomy of life each religious institute has the obligation and the right to establish their own provisions of proper law, including the fundamental code, namely the constitutions and other codes.

\footnotetext{
${ }^{109}$ Cf. Mensaje de Capitulo General a todos los religiosos de la Orden, in: Normae Capituli Generalis O.C.D. - 1973, 206-207.

${ }^{110} \mathrm{VC} 37$.
} 
The applicable Code of Canon Law specifies their aims, basic rules of their redacting and amending, as well as the competent authority to approve them. The works on the fundamental legislative text of the Law Proper of the Teresian Carmel commenced in 1967 and concluded in 1986 by the approval of The Constitutions and Norms. According to it the special role is given to the constitutions which not only protect the mission and identity of each religious institute, but has a considerable involvement in a continual revival of the religious life of each institute and its members. So that the proper law would fulfill its function, it needs to be better and better learnt and deeply considered, as well as suitably revised and adapted with respect to doctrinal and charismatic enhancement of certain issues, new signs of the times and new instructions of the Magisterium of the Church. A great help in this realm may be the post-council experiences of individual institutes in the thoroughgoing reform of their proper law, practical conclusions drawn from legislative works and the appropriate initial and continual formation.

SŁOWA KLUCzowe: instytut zakonny; Karmel Terezjański; prawo własne; kodeks fundamentalny; rewizja konstytucji; odnowa życia zakonnego

KEYwORDs: religious institute; Teresian Carmel; proper law; fundamental code; revision of constitutions; revival of religious life

\section{Nota o Autorze:}

O. Prof. DR hab. Wieslaw Kiwior OCD - kapłan Zakonu Karmelitów Bosych, profesor Wydziału Prawa Kanonicznego Uniwersytetu Kardynała Stefana Wyszyńskiego w Warszawie. W latach 2012-2016 prodziekan Wydziału; kierownik Katedry Kanonicznego Prawa Karnego, Administracyjnego i Procesowego; kierownik Zakładu kanonicznego Prawa Procesowego. Autor licznych publikacji naukowych. 